\title{
Intra-Arrest Administration of Cyclosporine and Methylprednisolone Does Not Reduce Postarrest Myocardial Dysfunction
}

\author{
Meshe Chonde $\mathbb{D}^{1},{ }^{1}$ Katharyn L. Flickinger, ${ }^{2}$ Matthew L. Sundermann, ${ }^{2}$ \\ Allison C. Koller, ${ }^{2}$ David D. Salcido, ${ }^{2}$ Cameron Dezfulian, ${ }^{3}$ \\ James J. Menegazzi, ${ }^{2}$ and Jonathan Elmer ${ }^{2,3}$ \\ ${ }^{1}$ UPMC Heart and Vascular Institute, Department of Cardiology, USA \\ ${ }^{2}$ University of Pittsburgh, Department of Emergency Medicine, USA \\ ${ }^{3}$ University of Pittsburgh, Department of Critical Care Medicine, USA \\ Correspondence should be addressed to Meshe Chonde; meshe.chonde@gmail.com
}

Received 5 March 2019; Accepted 13 May 2019; Published 11 June 2019

Academic Editor: Hideo Inaba

Copyright (C) 2019 Meshe Chonde et al. This is an open access article distributed under the Creative Commons Attribution License, which permits unrestricted use, distribution, and reproduction in any medium, provided the original work is properly cited.

Objective. To determine whether the administration of intra-arrest cyclosporine (CCY) and methylprednisolone (MP) preserves left ventricular ejection fraction (LVEF) and cardiac output (CO) after return of spontaneous circulation (ROSC). Methods. Eleven, $25-30 \mathrm{~kg}$ female swine were randomized to receive $10 \mathrm{mg} / \mathrm{kg} \mathrm{CCY}+40 \mathrm{mg} \mathrm{MP}$ or placebo, anesthetized and given a transthoracic shock to induce ventricular fibrillation. After 8 minutes, standard CPR was started. After two additional minutes, the experimental agent was administered. Animals with ROSC were supported for up to $12 \mathrm{~h}$ with norepinephrine as needed. Echocardiography was performed at baseline, and 1, 2, 6 and 12h post-ROSC. Analysis was performed using generalized estimating equations (GEE) after downsampling continuously sampled data to 5 minute epochs. Results. Eight animals (64\%) achieved ROSC after a median of 7 [IQR 5-13] min of CPR, 2 [ IQR 1-3] doses of epinephrine and 2 [IQR 1-5] defibrillation shocks. Animals receiving CCY +MP had higher post ROSC MAP (GEE coefficient $-10.2, \mathrm{P}=<0.01$ ), but reduced cardiac output (GEE coefficient $0.8, \mathrm{P}=<0.01$ ) compared to placebo. There was no difference in LVEF or vasopressor use between arms. Conclusions. Intra-arrest cyclosporine and methylprednisolone decreased post-arrest cardiac output and increased mean arterial pressure without affecting left ventricular ejection fraction.

\section{Introduction}

Each year, more than 500,000 cardiac arrests occur in the United States alone, and patient outcomes remain unacceptably poor [1]. Many patients that achieve return of spontaneous circulation (ROSC) develop subsequent hemodynamic instability, macro- and microcirculatory dysfunction, and end organ damage, a process that has been broadly described as the "postresuscitation syndrome" [2,3]. Significant left ventricular systolic and diastolic dysfunction during this period is common and has been termed postarrest myocardial dysfunction (PAMD) [4]. Observational studies have suggested an association between PAMD and worse patient outcomes $[5,6]$.

The pathogenesis of PAMD involves ischemiareperfusion injury, activation of the inflammatory cascade, and elevated levels of circulating catecholamines, all of which can worsen myocardial function $[2,7,8]$. The complexity of these interdependent pathways makes it unlikely that a single drug will be an effective therapy [9]. Rather, a cocktail of several agents with different targets may be necessary. One promising potential drug combination that targets multiple pathways thought to contribute to PAMD is cyclosporine (CCY) and methylprednisolone 
(MP). CCY blocks the mitochondrial permeability transition pore thereby preventing apoptosis and cell death [10] and improving contractility [11, 12], while MP decreases macrophage and leukocyte activation [13]. Both agents also decrease inflammatory cytokine production [10, 14-19].

Several preclinical studies have suggested that CCY $[10$, 15-18] or corticosteroids [20-22] attenuate PAMD when tested individually. However, these studies have measured cardiovascular function only briefly after return of spontaneous circulation (ROSC). Moreover, the effect of CCY and MP in combination has never been evaluated. Thus, we sought to examine the effect of combination treatment on longer-term myocardial function after ROSC. Our primary hypothesis was that $\mathrm{CCY}+\mathrm{MP}$ would reduce PAMD compared to placebo. Our secondary hypothesis was that $\mathrm{CCY}+\mathrm{MP}$ would decrease the inflammatory response compared to placebo.

\section{Methods}

All aspects of this study were reviewed and approved by the University of Pittsburgh Institutional Animal Care and Use Committee. The experiment was conducted in compliance with the NIH Guide for the Care and Use of Animals.

2.1. Animal Preparation. We sedated 11 female, mixed breed swine, weighing $25-35 \mathrm{~kg}$ with intramuscular ketamine $(10 \mathrm{mg} / \mathrm{kg})$ and xylazine $(4 \mathrm{mg} / \mathrm{kg})$. Once sedated, we placed a $20 \mathrm{~g}$ intravenous catheter in a peripheral ear vein, administered a $50 \mathrm{mcg} / \mathrm{kg}$ bolus of IV fentanyl and $5 \mathrm{mg}$ vecuronium, and intubated the trachea with direct laryngoscopy and a 50 cuffed endotracheal tube. We then initiated and titrated continuous infusions of intravenous fentanyl (starting dose $200 \mathrm{mcg} / \mathrm{kg} / \mathrm{hr}$ ) and midazolam (starting dose $4 \mathrm{mg} / \mathrm{hr}$ ) to maintain a surgical plane of anesthesia. We administered $1 \mathrm{~g}$ ceftriaxone and $500 \mathrm{mg}$ metronidazole to minimize risk of sepsis from instrumentation and periarrest translocation of gastrointestinal flora into the blood stream.

We ventilated the animals with an Ohmeda 7000 ventilator (Ohmeda, BOC Health Care, Madison, WI), initial tidal volumes of $10 \mathrm{~mL} / \mathrm{kg}$, respiratory rates of $12-16$ breaths per minute and $21 \%$ oxygen. We titrated respiratory rate to maintain an end-tidal carbon dioxide between 35 and $45 \mathrm{mmHg}$ (Zoll M series CCT capnometer) and inspired oxygen to maintain arterial oxygen saturations $>94 \%$. We used standard Lead II electrocardiogram (ECG) configuration for cardiac monitoring. We placed 9 French femoral arterial and venous introducer sheaths via direct cut-down. Through the arterial sheath, we introduced a micromanometer-tipped catheter (Millar, Inc. Houston TX) into the descending aorta. Through the venous sheath, we introduced a Continuous Cardiac Output (CCO) thermodilution catheter (Edwards LifeScience, Irvine CA) into the pulmonary artery. We confirmed proper positioning of the catheters by inspection of the hemodynamic pressure tracings. We used a Vigilance II monitor (Edwards LifeScience) to calculate cardiac output from the $\mathrm{CCO}$ catheter. We measured and recorded all physiological parameters continuously using LabChart (AD Instruments, Colorado Springs CO).
We randomized animals in permuted blocks of 2 and 4 to study agent or placebo. A third party not involved in the experiment made the allocation tables and placed treatment assignments in opaque envelopes. Prior to inducing ventricular fibrillation (VF), an unblinded laboratory technician in a different room opened the envelope corresponding to the experiment number and prepared two opaque syringes containing $10 \mathrm{mg} / \mathrm{kg}$ of cyclosporine A (Sandimmune, Novartis) in a $10 \mathrm{~mL}$ syringe and $40 \mathrm{mg}$ methylprednisolone (Solumedrol, Pfizer, NY) reconstituted in $5 \mathrm{~mL}$ normal saline, or two syringes with equal volumes of saline (placebo).

We recorded instrumentation time as the duration from the initial bolus of fentanyl until VF was induced. We attempt to standardize this interval by arresting the animal as close to 60 minutes as possible. We induced VF by delivering a $60 \mathrm{~Hz}$, $100 \mathrm{~mA}$, alternating current shock via external transthoracic electrodes for three seconds. We allowed 8 minutes of untreated VF, after which we began closed-chest CPR with a mechanical compression device LUCAS $^{\mathrm{TM}}$, JOLIFE AB, Sweden) at a rate of 100 compressions per minute.

After 2 minutes of compressions (10 minutes from VF induction), we administered study drugs or placebo, as well as $40 \mathrm{U}$ vasopressin, $1 \mathrm{mEq} / \mathrm{kg}$ sodium bicarbonate, and $0.1 \mathrm{mg} / \mathrm{kg}$ epinephrine. We continued compressions for another 3 minutes and then performed pulse and rhythm checks at 2-minute intervals. If the animal remained pulseless then epinephrine $0.015 \mathrm{mg} / \mathrm{kg}$ bolus was administered and repeated every 4 minutes as needed and CPR continued. If VF or ventricular tachycardia were noted, we attempted defibrillation with a single 150J biphasic shock. We continued CPR until ROSC or until 20 minutes without successful resuscitation.

2.2. Postresuscitation Management. Animals that achieved ROSC received standardized postresuscitation critical care for 12 hours, with echocardiography and blood sampling at predetermined time intervals. Briefly, we titrated fentanyl and midazolam to maintain a surgical plane of anesthesia. If mean arterial pressure (MAP) fell below $65 \mathrm{mmHg}$, we checked for predicted fluid responsiveness by assessing pulse pressure variation with the respiratory cycle. We bolused hypotensive, fluid responsive animals with $10 \mathrm{ml} / \mathrm{kg}$ Lactated Ringer's, which we repeated until the animal was normotensive or no longer fluid responsive. We treated hypotensive animals that were not fluid responsive with a continuous infusion of norepinephrine started at $0.1 \mathrm{mcg} / \mathrm{kg} / \mathrm{min}$, which we titrated to maintain MAP $>65 \mathrm{mmHg}$. We titrated the respiratory rate, oxygen concentration, and positive end expiratory pressure to maintain an arterial oxygen saturation of $94-98 \%$ and partial pressure of arterial carbon dioxide $35-40 \mathrm{mmHg}$. We euthanized animals that survived for the 12 hours after ROSC with a rapid IV bolus of $40 \mathrm{mEq}$ potassium chloride.

\subsection{Data Collection}

2.3.1. Transthoracic Echocardiography. We performed twodimensional echocardiography using a portable transthoracic echocardiogram (Vivid e GE) at baseline (prearrest) and at 1 , $2,3,6$, and 12 hours after ROSC. We obtained left ventricular 
parasternal long axis views as has been described previously [23]. Two board certified echocardiographers blinded to treatment allocation independently quantified left ventricular ejection fraction and we analyzed the mean of the two reviewers' interpretations.

2.3.2. Blood Gas and Biomarker Collection. We obtained samples of arterial blood at baseline, and then at 1, 2, 3, 6 , and 12 hours after ROSC. We analyzed baseline, 1-hour and 6-hour samples, an arterial blood gas analyzer (I-Stat, Heska Copr. Wakesha, WA). We also obtained plasma from samples at all time points by placing the blood in sterile ethylenediaminetetraacetic acid- (EDTA-) treated tubes (BD) that we centrifuged at $5000 \mathrm{rpm}$ for 5 minutes. We allocated plasma into $500 \mathrm{uL}$ vials that we kept at $-80^{\circ} \mathrm{C}$ until analysis. We used a Luminex multiplex analyzer to simultaneously measure concentrations of IL-1B, IL-4, IL-8, IL-10, and TNFalpha using porcine specific antibodies (EMD, Billerica, MA).

2.3.3. Hemodynamics. We measured MAP and $\mathrm{CO}$ as described above and recorded these continuous data at $100 \mathrm{~Hz}$ using LabChart (AD Instruments, Castle Hill, Australia). We downsampled MAP and CO to mean values in consecutive 5-minute epochs for repeated measures analysis.

2.3.4. Statistical Analysis. We summarized baseline data using descriptive statistics and reported medians with interquartile ranges. We compared repeated measures data using generalized estimating equations (GEEs) with an unstructured covariance matrix and robust standard errors to account for the small sample size of our pilot data. We performed all statistical analyses using Stata v14.2 (StataCORP, College Station, TX) and considered a p-value $<0.05$ to be statistically significant..

\section{Results}

Baseline parameters were well matched between groups (Table 1). Overall, eleven animals were randomized (5 to $\mathrm{CCY}+\mathrm{MP}$ and 6 to placebo). One animal randomized to cyclosporine had significant blood loss during femoral vascular access and did not undergo induction of VF or the experimental procedure. This animal was excluded from analysis. 8 animals achieved ROSC (3/4 cyclosporine and $5 / 6$ placebo). In the animals that achieved ROSC, LVEF decreased after arrest with a nadir at $3 \mathrm{~h}$ and subsequent return to baseline (Figure 1) but did not differ across treatment groups (GEE coefficient 6.4, $\mathrm{p}=0.34$ ). Postarrest $\mathrm{CO}$ also initially decreased from baseline (Figure 2) but was significantly higher in the placebo group compared to $\mathrm{CCY}+\mathrm{MP}$ (GEE coefficient $0.8, \mathrm{P}=<0.01$ ). In contrast, MAP was significantly higher in the $\mathrm{CCY}+\mathrm{MP}$ group compared to placebo (GEE coefficient for MAP -10.2, P = <0.01) with no difference in overall vasopressor requirements, (coefficient for vasopressor dose $0.2, \mathrm{P}=0.26$ ) (Figure 3 ). However, in the first 4 hours after ROSC, vasopressor requirements were substantially higher in the placebo arm $(\mathrm{P}<0.01)$. Levels of IL-1B, IL-8, IL-10, and TNF-alpha did not differ across

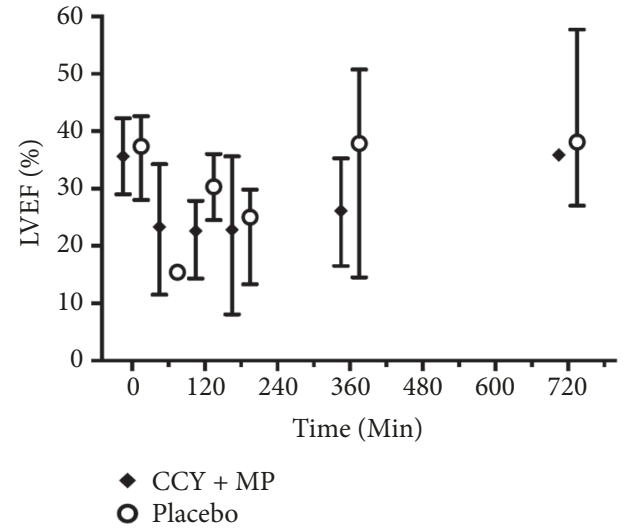

FIGURE 1: Left ventricular ejection fraction after ROSC. Median values with interquartile range error bars. $\mathrm{CCY}+\mathrm{MP}$, cyclosporine, and methylprednisolone; LVEF, left ventricular ejection fraction.

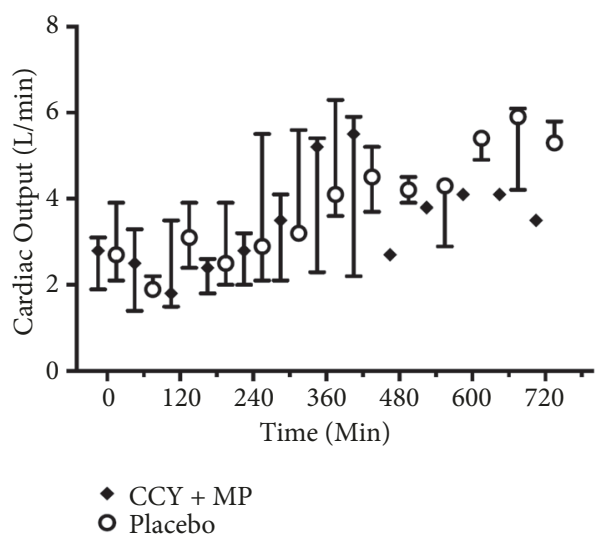

FIGURE 2: Cardiac output after ROSC. Median values with interquartile range error bars. $\mathrm{CCY}+\mathrm{MP}$, cyclosporine methylprednisolone.

treatment groups (see supplemental data). However, twentyseven percent of the plasma assays were contaminated by hemolysis/lipemia, limiting our ability to interpret these cytokine results.

\section{Discussion}

Postarrest myocardial dysfunction (PAMD) is common and worsens survival in those hospitalized after cardiac arrest. Despite its prevalence, effective strategies to attenuate the severity or duration of PAMD have remained elusive. We completed pilot work in a porcine model testing a novel drug combination targeting multiple cellular pathways believed to contribute to PAMD [7]. Our model itself was effective in that we observed a significant decrease in LV ejection fraction and high vasopressor requirements after ROSC in placebo animals. However, PAMD and shock were short-lived, with cardiovascular function returning to baseline by 6 hours after ROSC, and we observed no difference between drug and placebo arms in terms of myocardial function.

While our pilot work was not designed to let us test the independent effects of MP and CCY, we hypothesize that 
TABLE 1: Baseline Characteristics.

\begin{tabular}{|c|c|c|}
\hline & $\begin{array}{c}\mathrm{CCY}+\mathrm{MP} \\
(\mathrm{n}=5)\end{array}$ & $\begin{array}{c}\text { Control } \\
(n=6)\end{array}$ \\
\hline \multicolumn{3}{|l|}{ Hemodynamics } \\
\hline MAP (mmHg) & $88(88-96)$ & $85(74-92)$ \\
\hline $\mathrm{CO}(\mathrm{L} / \mathrm{min})$ & $2.8(1.9-3.1)$ & $2.7(2.1-3.9)$ \\
\hline \multicolumn{3}{|l|}{ Arrest characteristics } \\
\hline Weight (kg) & $25.0(24.4-27.5)$ & $24.2(23.8-25.4)$ \\
\hline CPR duration (min) & $7(5-7)$ & $7(5-13)$ \\
\hline Countershocks (\#) & $2(1.5-2)$ & $2(1-5)$ \\
\hline Epinephrine bolus (\#) & 2 & $2(1-3)$ \\
\hline \multicolumn{3}{|l|}{ Laboratory values } \\
\hline $\mathrm{pH}$ & $7.56(7.54-7.58)$ & $7.55(7.49-7.60)$ \\
\hline pCO2 (mmHg) & $38(37-40)$ & $32(31-38)$ \\
\hline pO2 (mmHg) & $89(88-240)$ & $93(77-107)$ \\
\hline $\mathrm{HCO} 3$ (meq/L) & $33.9(33.7-37.1)$ & $29.8(29.6-32.4)$ \\
\hline Sp02 (\%) & $98(98-100)$ & $99(96-99)$ \\
\hline $\mathrm{Na}(\mathrm{meq} / \mathrm{L})$ & $140(140-142)$ & $142(141-142)$ \\
\hline $\mathrm{K}(\mathrm{meq} / \mathrm{L})$ & $3.5(3.1-3.7)$ & $3.4(3.2-3.7)$ \\
\hline $\mathrm{Ca}(\mathrm{meq} / \mathrm{L})$ & $1.35(1.28-1.39)$ & $1.22(1.17-1.34)$ \\
\hline Glu (mmol/L) & $119(110-125)$ & $102(96-111)$ \\
\hline Hct & $25(25-27)$ & $25(24-26)$ \\
\hline \multicolumn{3}{|l|}{ Echocardiographic parameters } \\
\hline $\operatorname{LVEDD}(\mathrm{cm})$ & $3.5(3.3-3.6)$ & $2.9(2.9-3.0)$ \\
\hline $\operatorname{LVESD}(\mathrm{cm})$ & $2.7(2.4-2.9)$ & $2.1(2.0-2.2)$ \\
\hline Fractional Shortening (\%) & $23.9(19.3-28.5)$ & $26.9(24.3-32.8)$ \\
\hline LVEF (\%) & $35.6(29.0-42.3)$ & $37.5(328.0-42.6)$ \\
\hline
\end{tabular}

Data is shown as median (IQR). CO, cardiac output; Glu, glucose; Hct, Hematocrit; LVEDD, left ventricular end diastolic diameter; LVEF, left ventricular ejection fraction; LVESD, left ventricular end systolic diameter; MAP, mean arterial pressure; ROSC, return of spontaneous circulation

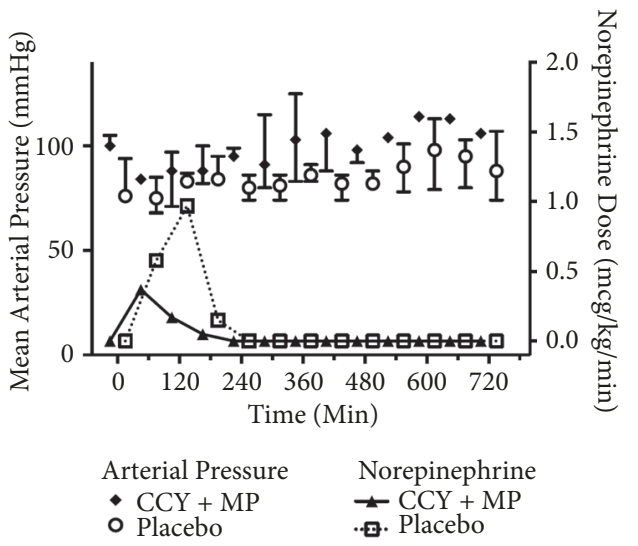

FIGURE 3: Mean arterial pressure and norepinephrine use after ROSC. Median values with interquartile range error bars. CCY $+\mathrm{MP}$, cyclosporine methylprednisolone.

the improvement in vascular tone we observed in the drug arm was due to the MP. Similar effects have been reported in clinical trials testing the utility of corticosteroids after cardiac arrest [24, 25]. Although CCY can cause hypertension [26], this is an effect noted in chronic use rather than an acute-phase effect. It may be that this improved vascular tone in the drug arm reduced cardiac output by increasing afterload. Alternatively, since LV systolic function and cardiac output are increased with norepinephrine [27-29], the trend towards increased vasopressor requirements in the placebo may actually have masked a lower LV ejection fraction and cardiac output. Interestingly, after 6 hours after ROSC, the $\mathrm{CCY}+\mathrm{MP}$ group had a decrease in cardiac output to baseline, while the placebo group continued to increase. As there was no further vasopressor use, noxious stimulus, or change in sedation this may represent the presence a "secondary insult" such as developing sepsis or delayed hemodynamic response to delayed cellular mediators of systemic inflammation.

Our finding that CCY+MP did not reduce PAMD differs from other preclinical studies that demonstrated an improvement or attenuation of postarrest or postcardiac bypass myocardial function in treatment with cyclosporine [11, 12, 15-18] but is consistent with the results of recent Phase III trials of CCY alone [30]. The dose of cyclosporine previously used in preclinical studies ranged from $2.5 \mathrm{mg} / \mathrm{kg}$ to $25 \mathrm{mg} / \mathrm{kg}$. We chose $10 \mathrm{mg} / \mathrm{kg}$, as Gill and colleagues [16] demonstrated in a newborn piglet model of asphyxial cardiac arrest that the attenuation of myocardial function and hemodynamics was greatest in those treated with cyclosporine $10 \mathrm{mg} / \mathrm{kg}$ 
compared to $25 \mathrm{mg} / \mathrm{kg}, 2.5 \mathrm{mg} / \mathrm{kg}$, or placebo. Additionally, cyclosporine in our study was administered intra-arrest, prior to ROSC, as suggested by Huang et al. [15], who demonstrated that delay in the administration of cyclosporine until postROSC did not yield significant benefit in attenuation of myocardial dysfunction. If not due to a different dose of medication or delay in administration then what could have played a role in the difference?

Perhaps, cyclosporine is not the right immunosuppressive to use. During the conduct of our work, the Phase III CYRUS trial [30], a multicenter randomized control trial of patients with a nonshockable cardiac arrest treated with intra-arrest cyclosporine demonstrated no improvement in survival, cardiac, or neurologic function. This trial has been criticized in that [9] median time from collapse to cyclosporine administration was at least 19 minutes. Additionally, patients received a dose of cyclosporine $2.5 \mathrm{mg} / \mathrm{kg}$ intra-arrest, which has been shown as a less effective dose in prior preclinical models [16] and is less than initial daily treatment dose for transplant patients [31], which may have also resulted in blunted effect. Our findings suggest that the neutral results in CYRUS may not be due only to the timing of drug administration or the dose as we observed no protective effects despite a 4 -fold higher dose.

From a mechanistic perspective, we found no significant differences in inflammatory cytokine levels across treatment arms. However, several of the samples were contaminated secondary to lipemia and were not suitable for analysis, which may have contributed to our neutral result. Due to our small sample size and limited specimens we were unable to analyze further, but it marks an area of interest for future study.

Our work has several important limitations. First, while our study was prospective, blinded, and the animal subjects randomized in 1:1 or 2:2 blocks, it was intended as pilot work to inform a future, larger study design. Therefore, our sample size was limited, potentially resulting in a Type II error. Although analyses of repeated measures can improve statistical power despite small sample sizes, ultimately our outcomes were still clustered within relatively few animals. Second, our overall ischemic insult during arrest and cardiopulmonary resuscitation was relatively short. This was by design to increase the number of animals achieving ROSC but may have resulted in less severe PAMD. Given the significant decrease in myocardial performance we observed in the hours after ROSC, we do believe that our model should have resulted in a sufficient effect size to test the efficacy of our drug combination but cannot be sure. Indeed, although profound, the PAMD observed in our model was relatively short-lived, unlike PAMD observed in the clinical setting which may persist for hours to days [5, 32]. Compared to our model, Kern and colleagues saw peak PAMD 2 hours after ROSC following 10-minute untreated VF and 6 hours after ROSC following 15-minute VF [33]. It may be that the combination of CCY+MP would have greater efficacy in a more severe model of injury with a greater systemic inflammatory and catecholamine response. Finally, to maintain transparency, we report the results of our biomarker assays which may have been limited by smaller sample size due to lipemia and hemolysis but represent an area for future investigation.

\section{Conclusion}

Our pilot work does not support pursuing combined intra-arrest administration of cyclosporine and methylprednisolone as a strategy to reduce postarrest myocardial dysfunction.

\author{
Abbreviations \\ CCY: Cyclosporine A \\ CO: Cardiac output \\ EDTA: Ethylenediaminetetraacetic acid \\ IL-1 $\beta$ : Interleukin- $1 \beta$ \\ IL-10: Interleukin-10 \\ IL-4: Interleukin-4 \\ IL-8: Interleukin-8 \\ LVEDD: Left ventricular end diastolic diameter \\ LVESD: Left ventricular end systolic diameter \\ LVEF: Left ventricular ejection fraction \\ MAP: $\quad$ Mean arterial pressure \\ MP: $\quad$ Methylprednisolone \\ ROSC: Return of spontaneous circulation \\ TNF- $\alpha$ : Tumor necrosis factor- $\alpha$.
}

\section{Data Availability}

The data used to support the findings of this study are included within the article.

\section{Conflicts of Interest}

The authors disclose that there are no potential conflicts of interest.

\section{Acknowledgments}

The funding for this project was provided by the internal grant from UPMC Heart and Vascular Institute and the NIH (5K12HL109068). Biomarker analysis was conducted at the UPCI Cancer Biomarkers Facility: Luminex Core Laboratory that is supported in part by award P30CA047904. None of the organizations mentioned played any role in the study design, collection, analysis, interpretation, or completion of the manuscript.

\section{Supplementary Materials}

Biomarker Levels after ROSC. Median values with interquartile range error bars. CCY $+\mathrm{MP}$, cyclosporine, and methylprednisolone; IL, interleukin; TNF, tumor necrosis factor. (Supplementary Materials)

\section{References}

[1] Mozaffarian, "Erratum: Heart Disease and Stroke Statistics 2015 Update: A Report From the American Heart Association (Circulation (2015) 131 (e29-e322) DOI:10.1161/CIR.0000000000000152)," Circulation, vol. 131, no. 24, p. e535, 2015. 
[2] RW. Neumar, JP. Nolan, and C. Adrie, "Post-cardiac arrest syndrome: epidemiology, pathophysiology, treatment, and prognostication. a consensus statement from the international liaison committee on resuscitation (american heart association, australian and new zealand council on resuscitation, european resuscitation council, heart and stroke foundation of canada, interamerican heart foundation, resuscitation council of asia, and the resuscitation council of southern africa); the american heart association emergency cardiovascular care committee; the council on cardiovascular surgery and anesthesia; the council on cardiopulmonary, perioperative, and critical care; the council on clinical cardiology; and the stroke council," Circulation, vol. 118, pp. 2452-2483, 2008.

[3] C. Adrie, M. Adib-Conquy, I. Laurent et al., "Successful cardiopulmonary resuscitation after cardiac arrest as a 'sepsis-like' syndrome," Circulation, vol. 106, no. 5, pp. 562-568, 2002.

[4] J. C. Jentzer, M. D. Chonde, A. Shafton et al., "Echocardiographic left ventricular systolic dysfunction early after resuscitation from cardiac arrest does not predict mortality or vasopressor requirements," Resuscitation, vol. 106, pp. 58-64, 2016.

[5] M. Ruiz-Bailén, E. Aguayo de Hoyos, S. Ruiz-Navarro et al., "Reversible myocardial dysfunction after cardiopulmonary resuscitation," Resuscitation, vol. 66, no. 2, pp. 175-181, 2005.

[6] W.-T. Chang, M. H.-M. Ma, K.-L. Chien et al., "Postresuscitation myocardial dysfunction: correlated factors and prognostic implications," Intensive Care Medicine, vol. 33, no. 1, pp. 88-95, 2007.

[7] J. C. Jentzer, M. D. Chonde, and C. Dezfulian, "Myocardial Dysfunction and Shock after Cardiac Arrest," BioMed Research International, vol. 2015, Article ID 314796, 2015.

[8] A. Chalkias and T. Xanthos, "Pathophysiology and pathogenesis of post-resuscitation myocardial stunning," Heart Failure Reviews, vol. 17, no. 1, pp. 117-128, 2012.

[9] L. B. Becker and P. B. Berger, "Cyclosporine for Reperfusion Injury After Cardiac Arrest: Too Little Too Late?” JAMA cardiology, vol. 1, no. 5, pp. 566-567, 2016.

[10] M. Cour, J. Loufouat, M. Paillard et al., "Inhibition of mitochondrial permeability transition to prevent the post-cardiac arrest syndrome: a pre-clinical study, European Heart Journal, vol. 32, no. 2, pp. 226-235, 2011.

[11] D. J. Hausenloy, S.-B. Ong, and D. M. Yellon, "The mitochondrial permeability transition pore as a target for preconditioning and postconditioning," Basic Research in Cardiology, vol. 104, no. 2, pp. 189-202, 2009.

[12] J. Knapp, J. Roewer, T. Bruckner, B. W. Böttiger, and E. Popp, "Evaluation of Cyclosporine a as a Cardio- and Neuroprotective Agent after Cardiopulmonary Resuscitation in a Rat Model," Shock, vol. 43, no. 6, pp. 576-581, 2015.

[13] D. Simon, L. Borradori, and H.-U. Simon, "Glucocorticoids in autoimmune bullous diseases: Are neutrophils the key cellular target?" Journal of Investigative Dermatology, vol. 133, no. 10, pp. 2314-2315, 2013.

[14] G. Varvarousi, A. Stefaniotou, D. Varvaroussis, and T. Xanthos, "Glucocorticoids as an Emerging Pharmacologic Agent for Cardiopulmonary Resuscitation," Cardiovascular Drugs and Therapy, vol. 28, no. 5, pp. 477-488, 2014.

[15] C.-H. Huang, M.-S. Tsai, C.-Y. Hsu et al., "Post-cardiac arrest myocardial dysfunction is improved with cyclosporine treatment at onset of resuscitation but not in the reperfusion phase," Resuscitation, vol. 82, supplement 2, pp. S41-S47, 2011.
[16] R. S. Gill, N. Manouchehri, J.-Q. Liu et al., "Cyclosporine treatment improves cardiac function and systemic hemodynamics during resuscitation in a newborn piglet model of asphyxia: A dose-response study," Critical Care Medicine, vol. 40, no. 4, pp. 1237-1244, 2012.

[17] M. Cour, M. Abrial, V. Jahandiez et al., "Ubiquitous protective effects of cyclosporine A in preventing cardiac arrest-induced multiple organ failure," Journal of Applied Physiology, vol. 117, no. 8, pp. 930-936, 2014.

[18] J. T. Strauch, D. Spielvogel, P. L. Haldenwang et al., "Cooling to 10 degrees $\mathrm{C}$ and treatment with cyclosporine a improve cerebral recovery following prolonged hypothermic circulatory arrest in a chronic porcine model," European Journal of CardioThoracic Surgery, vol. 27, pp. 74-80, 2005.

[19] P. J. Barnes, I. Adcock, M. Spedding, and P. M. Vanhoutte, "Antiinflammatory actions of steroids: molecular mechanisms," Trends in Pharmacological Sciences, vol. 14, no. 12, pp. 436-441, 1993.

[20] O. J. Liakopoulos, N. Teucher, C. Mühlfeld et al., "Prevention of TNFalpha-associated myocardial dysfunction resulting from cardiopulmonary bypass and cardioplegic arrest by glucocorticoid treatment," European Journal of Cardio-Thoracic Surgery, vol. 30, no. 2, pp. 263-270, 2006.

[21] J. Y. Duffy, D. P. Nelson, S. M. Schwartz et al., "Glucocorticoids reduce cardiac dysfunction after cardiopulmonary bypass and circulatory arrest in neonatal piglets," Pediatric Critical Care Medicine, vol. 5, no. 1, pp. 28-34, 2004.

[22] J. M. Pearl, D. M. Plank, K. M. McLean, C. J. Wagner, and J. Y. Duffy, "Glucocorticoids improve calcium cycling in cardiac myocytes after cardiopulmonary bypass," Journal of Surgical Research, vol. 167, no. 2, pp. 279-286, 2011.

[23] M. L. Riess, T. R. Matsuura, J. A. Bartos et al., "Anaesthetic Postconditioning at the Initiation of CPR Improves Myocardial and Mitochondrial Function in a Pig Model of Prolonged Untreated Ventricular Fibrillation," Resuscitation, vol. 85, no. 12, pp. 1745-1751, 2014.

[24] S. D. Mentzelopoulos, S. Malachias, C. Chamos et al., "Vasopressin, steroids, and epinephrine and neurologically favorable survival after in-hospital cardiac arrest: a randomized clinical trial," The Journal of the American Medical Association, vol. 310, no. 3, pp. 270-279, 2013.

[25] S. D. Mentzelopoulos, S. G. Zakynthinos, M. Tzoufi et al., "Vasopressin, epinephrine, and corticosteroids for in-hospital cardiac arrest," JAMA Internal Medicine, vol. 169, no. 1, pp. 1524, 2009.

[26] N. Robert, G. W. Wong, and J. M. Wright, "Effect of cyclosporine on blood pressure.," Cochrane Database of Systematic Reviews (Online), no. 1, p. CD007893, 2010.

[27] E. S. DeWitt, K. J. Black, R. R. Thiagarajan et al., "Effects of commonly used inotropes on myocardial function and oxygen consumption under constant ventricular loading conditions," Journal of Applied Physiology, vol. 121, no. 1, pp. 7-14, 2016.

[28] P. Perez, A. Kimmoun, V. Blime, and B. Levy, "Increasing mean arterial pressure in cardiogenic shock secondary to myocardial infarction: Effects on hemodynamics and tissue oxygenation," Shock, vol. 41, no. 4, pp. 269-274, 2014.

[29] A. Beurton, N. Ducrocq, T. Auchet et al., "Beneficial effects of norepinephrine alone on cardiovascular function and tissue oxygenation in a pig model of cardiogenic shock," Shock, vol. 46, no. 2, pp. 214-218, 2016. 
[30] L. Argaud, M. Cour, P. Dubien et al., "Effect of Cyclosporine in Nonshockable Out-of-Hospital Cardiac Arrest," JAMA Cardiology, vol. 1, no. 5, p. 557, 2016.

[31] A. Dipchand, B. McCrindle, and L. West, "Mycophenolate mofetil: clinical applications and pharmacokinetic monitoring in pediatric heart transplant recipients," The Journal of Heart and Lung Transplantation, vol. 22, no. 1, p. S134, 2003.

[32] I. Laurent, M. Monchi, J.-D. Chiche et al., "Reversible myocardial dysfunction in survivors of out-of-hospital cardiac arrest," Journal of the American College of Cardiology, vol. 40, no. 12, pp. 2110-2116, 2002.

[33] K. B. Kern, R. W. Hilwig, K. H. Rhee, and R. A. Berg, "Myocardial dysfunction after resuscitation from cardiac arrest: an example of global myocardial stunning," Journal of the American College of Cardiology, vol. 28, no. 1, pp. 232-240, 1996. 


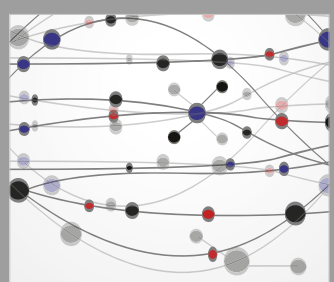

The Scientific World Journal
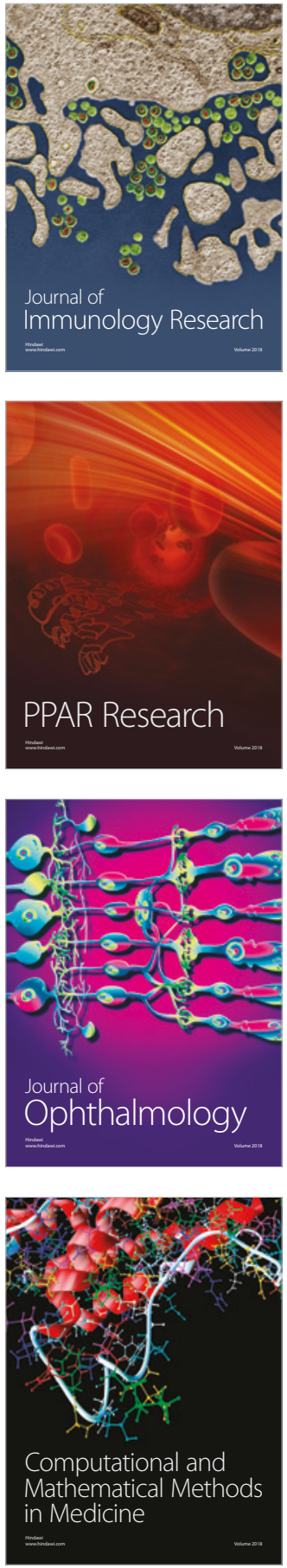

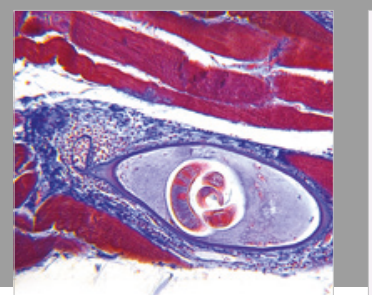

Gastroenterology Research and Practice

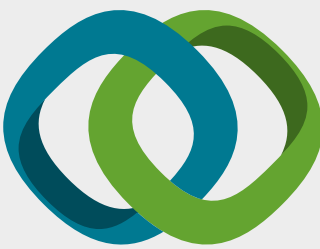

\section{Hindawi}

Submit your manuscripts at

www.hindawi.com
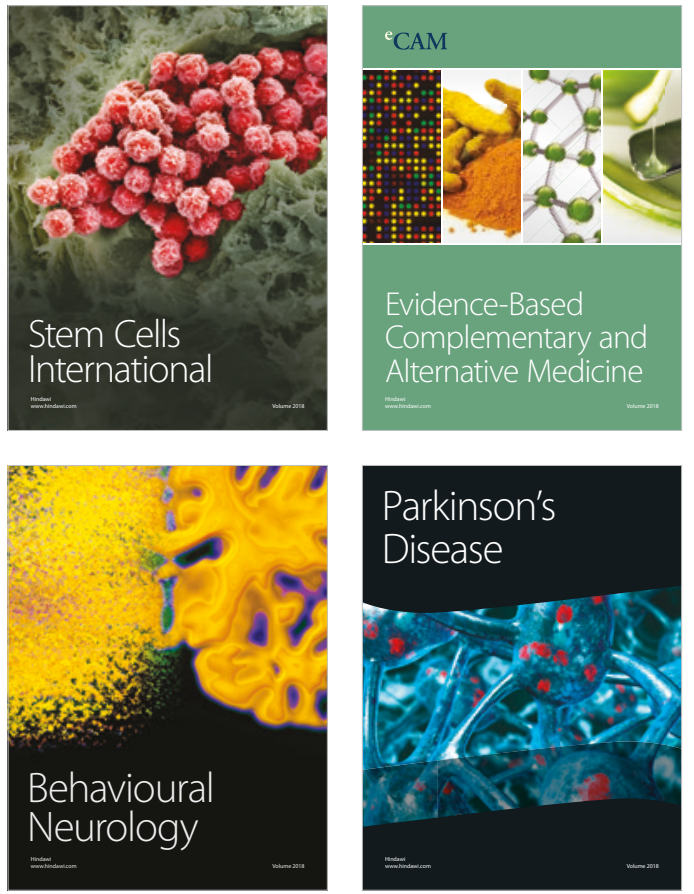

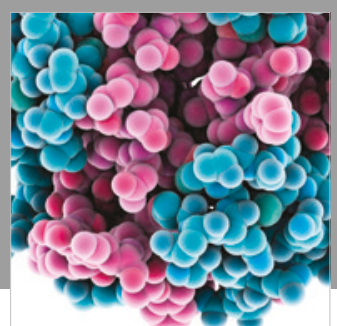

ournal of

Diabetes Research

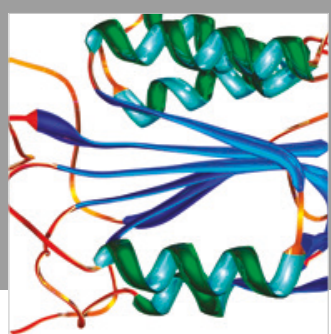

Disease Markers
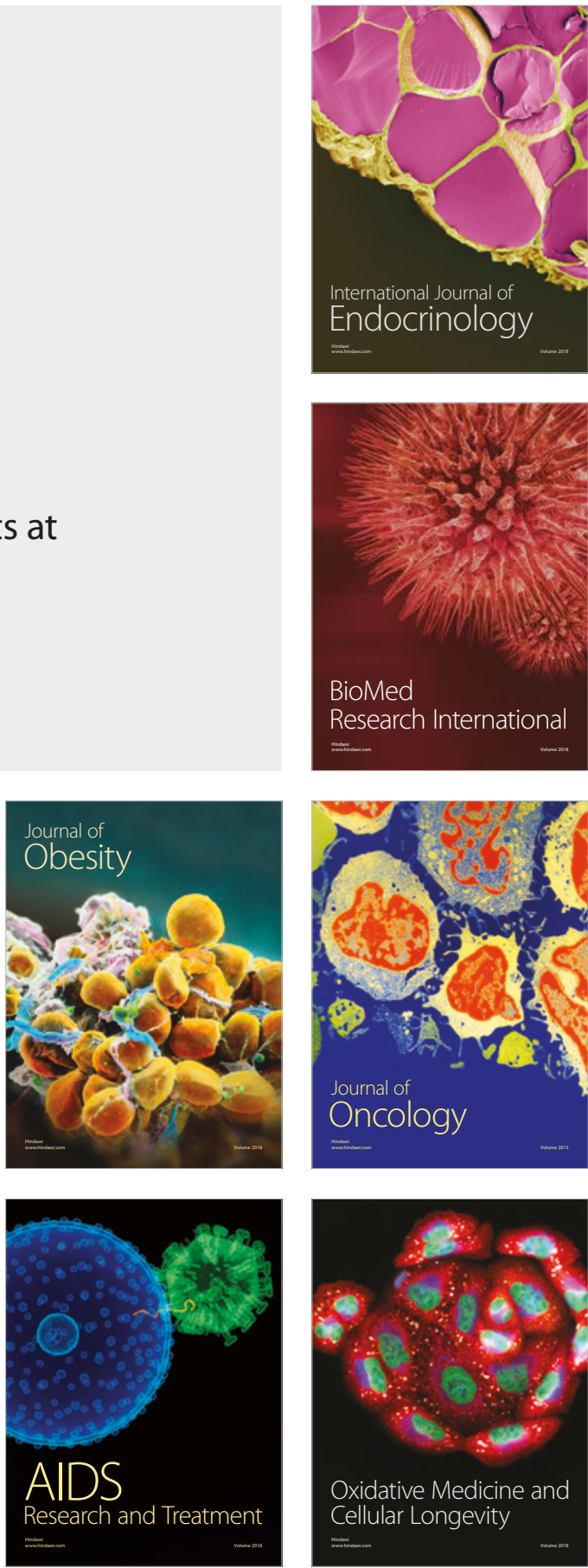